\title{
Fiabilidad y validez del cuestionario KIDSCREEN-52 para medir calidad de vida relacionada con la salud para población argentina de 8 a 18 años Reliability and validity of the KIDSCREEN-52 questionnaire to measure health related quality of life in the 8 to 18 year-old Argentinean population
}

\author{
Dra. Silvina Berra ${ }^{a, b}$, Mgt. Cristian Tebéc, ${ }^{, d}$ Dra. María Eugenia Esandie,f y \\ Dr. Carlos Carignanos
}

a. Consejo Nacional de Investigaciones Científicas y Técnicas (CONICET).

b. Escuela de Salud Pública, Facultad de Ciencias Médicas, Universidad

Nacional de Córdoba.

c. Agència d'Informació, Avaluació i Qualitat en Salut (AIAQS). Barcelona, España.

d. CIBER de

Epidemiología y Salud Pública (CIBERESP).

e. Instituto de Investigaciones Epidemiológicas, Academia Nacional de Medicina de Buenos Aires.

f. Área de Análisis Epidemiológico de los Determinantes de la Salud, Carrera de Medicina, Departamento de Economía, Universidad Nacional del Sur, Bahía Blanca.

g. Epidemiología Ambiental. Municipalidad de Bahía Blanca.

Correspondencia:

Dra. Silvina Berra:

silvinaberra@gmail.com

Conflicto de intereses: Ninguno que declarar.

Recibido: 22-2-2012

Aceptado: 22-8-2012

\section{RESUMEN}

Introducción. El cuestionario KIDSCREEN es una medida de la calidad de vida relacionada con la salud (CVRS). Permite discriminar niveles de salud o evaluar la eficacia de las intervenciones. El objetivo fue evaluar la fiabilidad y la validez de la versión argentina del cuestionario en población de 8-18 años de edad de la ciudad de Bahía Blanca.

Población y métodos. Estudio transversal en escuelas públicas y privadas durante el año 2008 mediante un muestreo estratificado por delegación. El cuestionario fue autoadministrado e incluyóel KIDSCREEN (52ítems) y preguntas sobre edad, sexo y el nivel socioeconómico (NSE) de la familia. Se analizó la fiabilidad mediante técnicas de análisis psicométrico clásicas, así como la teoría de respuesta al ítem. La estructura factorial fue analizada mediante un análisis factorial confirmatorio (AFC) y, la validez del constructo, comparando las puntuaciones medias de las dimensiones según edad, sexo y NSE.

Resultados. La mayoría de las dimensiones presentaron un alfa de Cronbach $>0,7$ y el $80 \%$ de los ítems, valores de infit o de outfit suficientes $(<0,8)$. El AFC mostró un adecuado ajuste de los datos a la estructura de diez dimensiones. Las medias de las dimensiones confirmaron puntuaciones menores en adolescentes que en niños/as y menores en mujeres que en varones, como se esperaba en varias dimensiones de la CVRS

Conclusiones. La versión argentina del KIDSCREEN-52 presentó una aceptable fiabilidad y validez, por lo que puede recomendarse su utilización en población argentina de 8-18 años. Palabras clave: salud infantil, adolescentes, calidad de vida, cuestionarios, validez de las pruebas.

http:/ /dx.doi.org/10.5546/aap.2013.29

\section{INTRODUCCIÓN}

El concepto de calidad de vida relacionada con la salud (CVRS) refiere a una percepción subjetiva de la capacidad para realizar aquellas funciones que son importantes para el individuo, influenciado por su estado de salud. Inicialmente, estos conceptos hacían referencia a las dimensiones físicas, psicológicas y sociales de la salud, ${ }^{1}$ pero con el tiempo se fue diversificando su multidimensionalidad hacia aspectos del bienestar o el funcionamiento en los otros ámbitos. Así, la declaración de la CVRS puede reflejar efectos de los procesos de salud-enfermedad o de factores que influyen sobre la salud, permite así una medida común para evaluar a personas con diferentes problemas de salud o discapacidad y puede ser indicadora de eficacia de tratamientos. ${ }^{2,3}$

Un atributo fundamental de las medidas de la CVRS es tener en cuenta la perspectiva de la propia persona. Actualmente existen herramientas que permiten obtener respuestas directas de niños, niñas y adolescentes sobre aspectos relevantes y específicos de su etapa vital, como las relaciones con amigos y familiares, que los propios niños y niñas señalan como el aspecto más importante de su salud. ${ }^{4}$

Los cuestionarios de CVRS para población infantil y adolescentes son ya un centenar, muchos de ellos evaluados en varios aspectos de su validez y fiabilidad, ${ }^{5}$ por lo que están en condiciones de ser utilizados en investigación, en la práctica clínica o en otro tipo de evaluaciones de intervenciones sanitarias. Existen versiones autoadministrables desde los 4 años y versiones para informadores indirectos que abarcan desde los 0 a los 18 años de 
edad. ${ }^{5}$ La mayoría de estos instrumentos fueron desarrollados en América del Norte o Europa y, entre las versiones que se encuentran en castellano, predominan las que han sido adaptadas para la población española. ${ }^{6}$ En América latina, ${ }^{7}$ y particularmente en la Argentina, se realizaron adaptaciones de estos cuestionarios y algunas de estas versiones han comenzado a implementarse tanto en el ámbito clínico como en el de la salud pública con distintos propósitos. ${ }^{8-10}$

El cuestionario KIDSCREEN ${ }^{11}$ se desarrolló por medio de una metodología rigurosa acorde a las recomendaciones internacionalmente consensuadas por un grupo de expertos en CVRS. ${ }^{12,13}$ Los ítems que componen el cuestionario fueron producidos a partir de grupos focales con niños, niñas y adolescentes de 8 a 18 años de varios países europeos. Luego fueron clasificados en dimensiones de la CVRS por expertos y probados en entrevistas con niños de las mismas edades para seleccionar aquellas preguntas, períodos temporales y escalas de respuesta que fueran mejor comprendidos por ellos. ${ }^{4}$ La selección final de ítems se realizó mediante análisis basados en la teoría psicométrica clásica y la teoría de la respuesta al ítem, de modo que la versión definitiva del cuestionario cumpliera con las propiedades métricas esperadas y no presentara sesgos por edad, sexo o país. ${ }^{14}$ En la Argentina se efectuó un proceso de adaptación transcultural ${ }^{15}$ para obtener un instrumento equivalente a la versión original europea. El método incluyó traducción directa e inversa con participación de lingüistas profesionales, entrevistas cognitivas con personas de diferentes edades, sexo y nivel socioeconómico para analizar la adecuación cultural; y para comprobar la equivalencia semántica entre la versión argentina y la original. ${ }^{10} \mathrm{El}$ objetivo del presente trabajo fue evaluar la fiabilidad y la validez de la versión argentina del cuestionario KIDSCREEN en una muestra representativa de la población escolarizada de 8 a 18 años de edad de la ciudad de Bahía Blanca.

\section{MÉTODOS}

Estudio de corte transversal realizado en escuelas públicas y privadas de la ciudad de Bahía Blanca durante el año 2008. La población elegible estuvo constituida por niños y adolescentes de ambos sexos, de 8 a 18 años de edad, que concurrían a estos establecimientos. Para el diseño de la muestra se utilizaron los datos correspondientes a la matrícula escolar provistos por la Dirección General de Escuelas ( $n=51$ 487).

La selección de la muestra se realizó mediante un muestreo por conglomerados en dos etapas. Las unidades de muestreo de la primera etapa fueron los establecimientos educativos estratificados por delegación y las de la segunda, los cursos dentro de los establecimientos seleccionados. El tamaño de la muestra fue calculado considerando un intervalo de confianza del $95 \%$ y un error máximo estimado para los parámetros de $0,02(2 \%)$. Se estimó una tasa de no respuesta del $20 \%$ y un error atribuible al diseño complejo de 3 deff (diferencias en la varianza respecto de un muestreo aleatorio simple), obteniendo un número necesario de casos de 3920 escolares. La unidad elemental fue el niño o adolescente seleccionado a partir del muestreo. Únicamente se incluyó a aquellos escolares cuyos padres dieron su consentimiento y autorizaron la participación de sus hijos en el estudio.

Se utilizó la versión argentina del cuestionario KIDSCREEN-52, que contiene 10 dimensiones recabadas mediante 52 ítems (Tabla 1), adecuado

TABLA 1. Dimensiones del instrumento KIDSCREEN, aspectos incluidos y número de ítems en cada una

- Bienestar físico: actividad física, sensación de energía y de estar físicamente en forma (5 ítems).

- Bienestar psicológico: emociones positivas y satisfacción con la vida (6 ítems).

- Estado de ánimo y emociones: experiencias negativas, estados depresivos y las sensaciones de estrés (7 ítems).

- Autopercepción: percepción de la apariencia física e imagen corporal, y la satisfacción relacionada con ellas (5 ítems).

- Autonomía: oportunidades percibidas para realizar actividades en el tiempo libre (5 ítems).

- Relación con los padres y vida familiar: atmósfera familiar (6 ítems).

- Apoyo social y relación con los pares: relación con los/as compañeros/as (6 ítems).

- Ambiente escolar: percepciones acerca de la propia capacidad cognitiva y de concentración, además de sensaciones acerca de la escuela (6 ítems).

- Aceptación social (bullying): sensación de rechazo de los/las compañeros/as (3 ítems).

- Recursos económicos: percepción sobre la capacidad financiera de la familia (3 ítems). 
para niños, niñas y adolescentes de 8 a 18 años. El cuestionario fue autoadministrado. Las respuestas a los ítems se categorizan en una escala de tipo Likert de 5 opciones, que evalúa la frecuencia o la intensidad del atributo con un período recordatorio de una semana en la mayoría de preguntas. A partir de las respuestas se calcularon las puntuaciones medias de cada dimensión, las que se estandarizaron a una media de 50 y una desviación estándar de 10, según las recomendaciones del grupo que desarrolló la versión original. El cuestionario utilizado incluía también la edad, el sexo y el nivel socioeconómico (NSE) de la familia de cada participante. El NSE se determinó a partir de la escala de bienestar económico familiar ( $F a-$ mily Affluence Scale, FAS), un índice creado a partir del número de automóviles y computadoras que posee la familia, la existencia de habitación propia e individual y haber salido de vacaciones con la familia en los últimos 12 meses. ${ }^{16}$ La escala se construye en 7 categorías (desde 0, el NSE inferior, hasta 7 , el más elevado) y se recodifica en 3 grupos: NSE bajo (0-3), intermedio (4-5) y elevado $(6-7){ }^{17}$

Cuatro personas, que fueron entrenadas para realizar la prueba piloto y ejecutar el trabajo de campo, presentaron el estudio en cada aula y resolvieron las dudas de los escolares mientras respondían la encuesta.

La consistencia interna de los ítems respecto de la dimensión correspondiente se analizó mediante el coeficiente alfa de Cronbach, ${ }^{18}$ para el cual se estableció como aceptable un valor mínimo de 0,7 . Se analizaron los valores perdidos y el efecto piso y techo, representados por el porcentaje de individuos que puntúan el mínimo y el máximo, respectivamente, de todos los valores posibles de cada escala, existiendo la convención de que un valor de hasta el $15 \%$ es aceptable para los tres indicadores. ${ }^{19}$

Sobre la base de la teoría de la respuesta al ítem y los antecedentes de las versiones originales se esperaba comprobar la unidimensionalidad de cada ítem y su independencia local. La unidimensionalidad es una propiedad que se cumple cuando cada uno de los ítems apunta o explica un único rasgo o habilidad, mientras que la independencia local se cumple cuando la probabilidad de responder positivamente a un ítem no condiciona la respuesta de otro ítem en aquellas personas con el mismo nivel en el rasgo. ${ }^{20}$ Estas propiedades se analizaron siguiendo el modelo teórico de crédito parcial (PCM, de partial credit model), que pertenece a la familia de los modelos Rasch. Para evaluar el comportamiento de los ítems en estos modelos se utilizaron los estadísticos Infit y Outfit, y se consideró que un ítem era adecuado cuando el estadístico Infit/Outfit se encontrara entre 0,8 y 1,2. ${ }^{21}$

Finalmente, un análisis factorial confirmatorio permitió evaluar el ajuste de los datos a la estructura preestablecida entre los ítems del cuestionario y sus dimensiones. La bondad de ajuste de los datos se midió con los índices de ajuste comparativo (CFI, de comparative fit index; criterio mínimo $\geq 0,9$ ), el índice de ajuste normalizado (NFI, de normed fit index; criterio mínimo $\geq 0,9)$ y la raíz cuadrada media del error de aproximación (RMSEA, de root mean square error of approximation; criterio mínimo $\leq 0,06) .{ }^{22}$

Para evaluar la validez del constructo se compararon las puntuaciones medias de las dimensiones del KIDSCREEN-52 según la edad, el sexo y la escala FAS; y se estimó la magnitud del efecto mediante el cálculo de las diferencias de medias estandarizadas (effect size). Se estableció que diferencias entre 0,2 y 0,5 serían mínimas, de 0,51 a 0,8 serían moderadas y mayores a 0,8 serían relevantes. ${ }^{23}$ Los análisis estadísticos se realizaron con el programa SPSS versión 15 y el modelo Rasch y el análisis factorial confirmatorio con el programa $\mathrm{R}$ versión 2.12.2.

\section{RESULTADOS}

El 85,7\% ( $n=4488)$ de los 5236 escolares seleccionados inicialmente completó la encuesta. Los principales motivos de no respuesta fueron la no asistencia el día de la administración $(n=509)$ y la falta de autorización para participar por parte de los padres $(n=227)$. Ochenta y seis casos fueron eliminados por tener menos de 8 o más de 18 años, quedando la muestra constituida por 4402 escolares, de los cuales el 43,6\% tenía entre 8 y 12 años, mientras que el 56,4\% tenía entre 13 y 18 , con distribución similar entre mujeres y varones. El 35,1\% de la muestra fue clasificado en el nivel socioeconómico bajo, el $44,4 \%$ en el nivel medio y el 20,5\% en el nivel alto (Tabla 2).

La cantidad de casos perdidos por falta de respuesta en ítems de una escala no alcanzó al 5\% en ninguna de ellas (Tabla 3). El efecto piso y techo fue mínimo en todas las dimensiones, salvo en la dimensión aceptación social, en la que el efecto techo alcanzó el 31,4\%. La mayoría de las dimensiones presentaron una consistencia interna mayor de 0,7 , excepto las de autopercepción $(\mathrm{alfa}=0,68)$ y aceptación social $(\mathrm{alfa}=0,64)$.

En 37 de 52 ítems se comprobó la unidimensionalidad e independencia local. La Figura 1 es un 
ejemplo de los resultados obtenidos en un ítem de la dimensión de bienestar físico. Los ítems que mostraron valores de infit o de outfit menores de 0,8 afectaron especialmente a las dimensiones de recursos económicos y rechazo social, donde 2 y 3 ítems, respectivamente, presentaron esa limitación. La Figura 2 presenta un mapa persona-ítem de la misma dimensión. Los datos sobre el resto de ítems y dimensiones se encuentran a disposición de los lectores.
Los resultados del análisis factorial confirmatorio mostraron un adecuado ajuste de los datos a la estructura de diez dimensiones. Los estadísticos asociados con la bondad de ajuste del modelo presentaron resultados aceptables $(\mathrm{NFI}=0,89$; CFI= 0,90; índice RMSEA=0,042).

Finalmente, las puntuaciones medias del KIDSCREEN confirmaron las diferencias esperadas según la edad y el sexo. Los chicos y chicas en la adolescencia presentaron puntuaciones me-

TABla 2. Frecuencia de sexo, nivel socioeconómico y nivel de estudios de la madre y del padre por grupos de edad y en toda la muestra

\begin{tabular}{|c|c|c|c|c|c|c|}
\hline & \multicolumn{2}{|c|}{ Niños } & \multicolumn{2}{|c|}{ Adolescentes } & \multicolumn{2}{|c|}{ Toda la muestra } \\
\hline & $\mathbf{n}$ & $\%$ & $\mathrm{n}$ & $\%$ & n & $\%$ \\
\hline \multicolumn{7}{|l|}{ Sexo } \\
\hline Femenino & 1024 & 53,3 & 1335 & 53,8 & 2359 & 53,6 \\
\hline Masculino & 897 & 46,7 & 1145 & 46,2 & 2042 & 46,4 \\
\hline Total & 1921 & 100,0 & 2480 & 100,0 & 4401 & 100,0 \\
\hline \multicolumn{7}{|l|}{ Nivel socioeconómico* } \\
\hline Bajo & 561 & 30,0 & 950 & 39,0 & 1511 & 35,1 \\
\hline Medio & 931 & 49,8 & 983 & 40,4 & 1914 & 44,4 \\
\hline Alto & 378 & 20,2 & 503 & 20,6 & 881 & 20,5 \\
\hline Total & 1870 & 100,0 & 2436 & 100,0 & 4306 & 100,0 \\
\hline \multicolumn{7}{|l|}{ Nivel de estudios de la madre* } \\
\hline No fue a la escuela & 77 & 4,3 & 39 & 1,6 & 116 & 2,8 \\
\hline Primario & 348 & 19,6 & 507 & 20,8 & 855 & 20,3 \\
\hline Secundario & 667 & 37,5 & 1078 & 44,2 & 1745 & 41,4 \\
\hline Terciario o universitario & 687 & 38,6 & 813 & 33,4 & 1500 & 35,6 \\
\hline Total & 1779 & 100,0 & 2437 & 100,0 & 4216 & 100,0 \\
\hline \multicolumn{7}{|l|}{ Nivel de estudios del padre* } \\
\hline No fue a la escuela & 91 & 5,3 & 53 & 2,2 & 144 & 3,5 \\
\hline Primario & 322 & 18,8 & 584 & 24,5 & 906 & 22,1 \\
\hline Secundario & 644 & 37,6 & 983 & 41,3 & 1627 & 39,8 \\
\hline Terciario o universitario & 655 & 38,3 & 759 & 31,9 & 1414 & 34,6 \\
\hline Total & 1712 & 100,0 & 2379 & 100,0 & 4091 & 100,0 \\
\hline
\end{tabular}

*Valor $\mathrm{p}<0,05$ en la prueba de la ji al cuadrado $\left(\chi^{2}\right)$ para las diferencias de frecuencias entre grupos de edad.

TABLA 3. Propiedades métricas de la versión argentina del KIDSCREEN-52

\begin{tabular}{|c|c|c|c|c|c|c|c|}
\hline & \multirow[b]{2}{*}{$\begin{array}{l}\text { Nros. de } \\
\text { ítems }\end{array}$} & \multirow[b]{2}{*}{$\begin{array}{c}\text { Valores } \\
\text { perdidos }(\%)^{*}\end{array}$} & \multirow[b]{2}{*}{$\begin{array}{c}\text { Efecto } \\
\text { piso (\%) }\end{array}$} & \multirow[b]{2}{*}{$\begin{array}{c}\text { Efecto } \\
\text { techo }(\%)\end{array}$} & \multirow[b]{2}{*}{$\begin{array}{l}\text { Alfa de } \\
\text { Cronbach }\end{array}$} & \multicolumn{2}{|c|}{ Análisis Rash: PCM } \\
\hline & & & & & & $\begin{array}{c}\begin{array}{c}\text { Infit } \\
\text { (min-máx) }\end{array} \\
\end{array}$ & $\begin{array}{c}\text { Outfit } \\
\text { (min-máx) }\end{array}$ \\
\hline Bienestar físico & 5 & 4,5 & 0,1 & 0 & 0,77 & $0,68-1,01$ & $0,69-1,00$ \\
\hline Bienestar emocional & 6 & 2,0 & 0,05 & 12,3 & 0,85 & $0,79-1,03$ & $0,78-1,04$ \\
\hline Estado de ánimo & 7 & 4,2 & 0,3 & 6,1 & 0,84 & $0,72-1,12$ & $0,70-1,13$ \\
\hline Autopercepción & 5 & 3,3 & 0,2 & 16,5 & 0,82 & $0,78-1,09$ & $0,62-1,07$ \\
\hline Autonomía & 5 & 2,5 & 0,3 & 18,9 & 0,88 & $0,73-0,99$ & $0,69-0,97$ \\
\hline Relación con los padres y vida familiar & 6 & 3,4 & 0,05 & 15,4 & 0,68 & $0,74-0,89$ & $0,72-0,93$ \\
\hline Apoyo social y relación con pares & 6 & 4,5 & 0,5 & 13,5 & 0,81 & $0,76-0,92$ & $0,76-0,93$ \\
\hline Ambiente escolar & 6 & 3,4 & 0,2 & 7,4 & 0,86 & $0,76-0,95$ & $0,74-0,98$ \\
\hline Aceptación social (bullying) & 3 & 2,6 & 0,6 & 31,4 & 0,65 & $0,66-0,78$ & $0,59-0,73$ \\
\hline Recursos económicos & 3 & 2,7 & 2,1 & 13,1 & 0,83 & $0,64-0,82$ & $0,65-0,81$ \\
\hline
\end{tabular}

* Porcentaje de casos en los que no se pudo calcular la dimensión por falta de respuesta de más del 50\% de los ítems. 
FIGURA 1. Curva característica de un ítem de la dimensión de bienestar físico

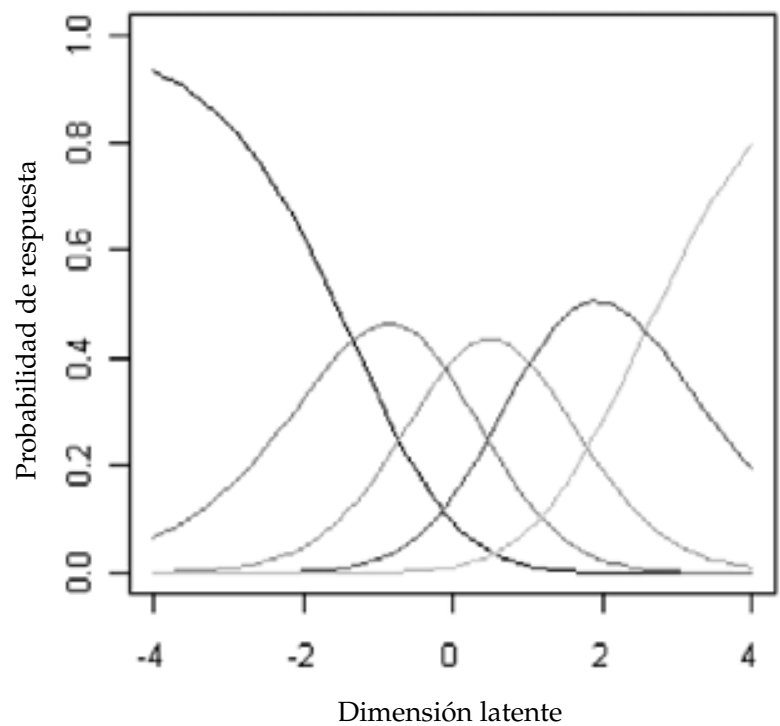

El eje de la X es valor de la dimensión latente (por ejemplo: bienestar físico): el eje de las Y es la probabilidad de respuesta. Cada curva representa la distribución de los valores de probabilidad de respuesta de los individuos en cada una de las categorías del ítem analizado (en este caso: “¿Te sentiste bien y físicamente en forma?"). La primera curva de la izquierda representa la categoría más baja ("nada") y muestra una distribución de sus valores principalmente en la parte baja de la dimensión latente (valores $<0$ ). La última curva de la derecha representa la categoría máxima ("muchísimo") con una distribución de sus valores principalmente en la parte alta de la dimensión latente (valores $>0$ ). Las demás curvas representan los valores de las categorías intermedias y sus valores esperados (parte alta de la curva), siguen el orden marcado por las categorías de respuesta adyacentes.

FIGURA 2. Mapa persona-ítem de la dimensión de bienestar físico

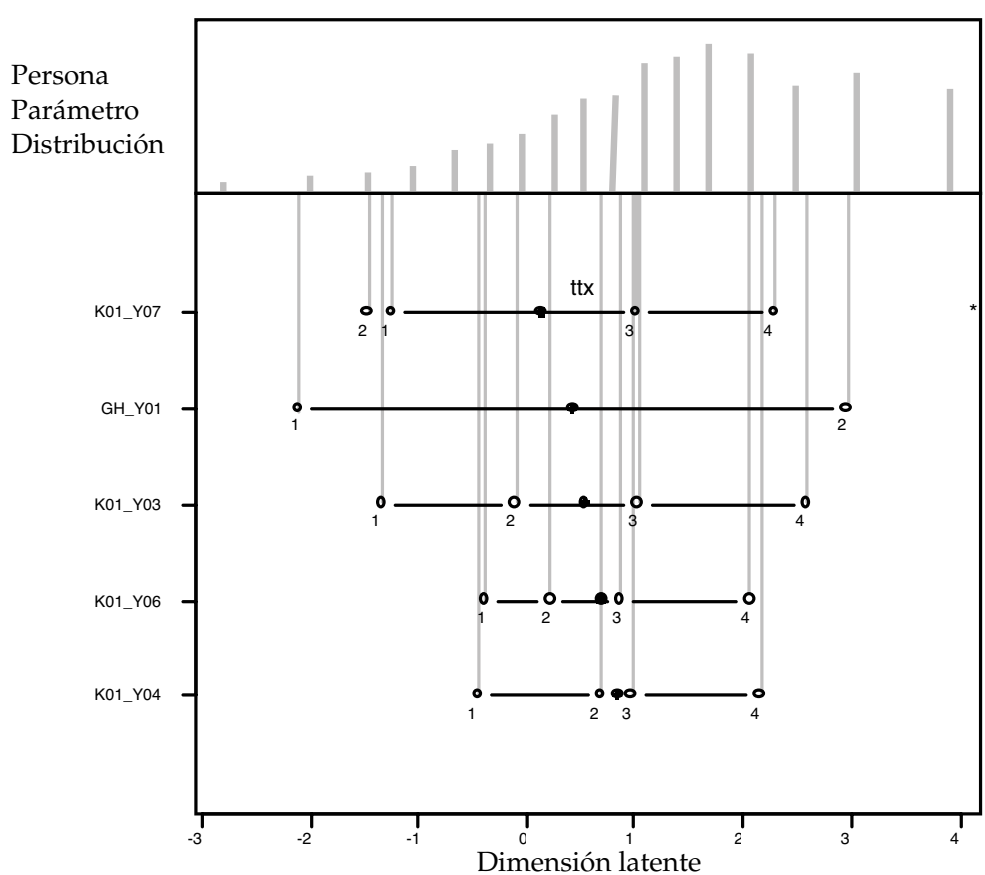

En la parte superior de la figura, el eje de la X representa los valores de la dimensión latente (bienestar físico) y el eje de las Y, el número de niños y niñas. Las columnas son el número de niños y niñas con una puntuación determinada en la dimensión latente. La situación óptima para esta dimensión es una curva acampanada centrada en valores medianos altos y con una larga cola para los valores bajos (hacia la izquierda). La parte inferior de la figura tiene el mismo eje X y no hay eje Y. Los puntos representan la categoría de respuesta de un ítem. De forma horizontal se presentan cada uno de los ítems de la dimensión. El valor de cada punto en el eje $\mathrm{X}$ muestra en qué valor de la puntación de la dimensión latente se ubica cada una de las categorías de respuesta de cada ítem. La situación óptima, para una mayor capacidad explicativa de los ítems de la dimensión, es que abarquen un amplio intervalo de valores de la puntuación latente. 
nores en la mayoría de las dimensiones, con un tamaño del efecto (TE) importante en Bienestar físico $(\mathrm{TE}=0,8)$, Bienestar psicológico $(\mathrm{TE}=0,8)$ y Ambiente escolar $(\mathrm{TE}=1,1)$, mientras que en las dimensiones de Apoyo social y Relación con amigos, las puntuaciones fueron más bajas en los menores de 12 años, pero con tamaños del efecto mínimos (Tabla 4). En cuanto a las puntuaciones medias por sexo (Tabla 4), los niños presentaron puntuaciones superiores a las niñas en cuatro dimensiones con tamaños del efecto mínimos $(\mathrm{TE}=0,2-0,4)$, pero las niñas tuvieron puntuaciones promedio más elevadas en la dimensión de Ambiente escolar $(\mathrm{TE}=-0,2)$.

\section{DISCUSIÓN}

En la Argentina aún existen pocos cuestionarios para evaluar la CVRS en población infantil y adolescente. ${ }^{7}$ El presente trabajo muestra que la fiabilidad y validez del KIDSCREEN adaptado para población argentina son buenas, lo que constituye una sólida base científica para apoyar su empleo a nivel local y es fundamental para la comparación de resultados en el contexto regional e internacional. Asimismo, estos resultados contribuyen a reducir la brecha de conocimiento sobre las propiedades métricas de instrumentos para su uso en poblaciones de habla castellana.

La consistencia interna y el ajuste de los ítems que componen las dimensiones de la medida de la CVRS al modelo Rash obtuvieron valores muy aceptables en la mayoría de las dimensiones. Estos resultados confirman que cada ítem de la versión adaptada proporciona información sobre diferentes rasgos de la CVRS de un niño, niña o adolescente de manera apropiada y que cada dimensión ofrece un valor de la salud percibida bajo un supuesto multidimensional. Algunos coeficientes de fiabilidad resultaron algo más bajos que los obtenidos por las versiones originales, ${ }^{14,24}$ pero se encuentran en niveles deseables, algo que puede considerarse un excelente resultado para un instrumento que ha sido desarrollado en otra población y que tras un riguroso proceso de adaptación cultural se muestra fiable también para la población argentina. Los resultados dan cuenta así de la equivalencia de medida entre la versión argentina y las originales europeas.

De manera similar a lo observado con la versión original del KIDSCREEN y otros instrumentos que miden CVRS, el análisis de validez del constructo mostró que la versión KIDSCREEN adaptada en población argentina es capaz de discriminar aspectos diferenciales de la salud según edad y sexo, como se espera en este lapso de transición desde la infancia hasta la adultez en que se observan cambios biológicos y psicosociales. Las niñas refirieron un bienestar físico y autopercepción algo peor que los niños, pero obtuvieron mejores puntuaciones en algunas de las dimensiones sociales, como ambiente escolar. También se comprobó que niños y niñas declaran una mejor CVRS que los y las adolescentes, excepto en la dimensión de aceptación social. El bienestar físico, el bienestar emocional y el ambiente escolar son las dimensiones que mostraron puntuaciones más bajas en los adolescentes de ambos sexos. Estos resultados de la CVRS según edad y sexo son consistentes a los observados en aplicaciones de la versión original del KIDSCREEN en otras poblaciones de niños y adolescentes.

Entre las limitaciones del presente estudio podría citarse el hecho de haberse realizado en una única ciudad de la República Argentina. Sin em-

TABLA 4. Puntuaciones promedio y desvío estándar de las dimensiones del KIDSCREEN por grupos de edad y sexo

\begin{tabular}{|c|c|c|c|c|c|c|c|c|c|c|}
\hline \multirow[t]{2}{*}{ Dimensiones CVRS } & \multicolumn{2}{|c|}{ Niños } & \multicolumn{2}{|c|}{ Adolescentes } & \multicolumn{3}{|c|}{ Sexo masculino } & \multicolumn{2}{|c|}{ Sexo femenino } & \multirow{2}{*}{$\begin{array}{c}\text { Tamaño del } \\
\text { efecto (d) }\end{array}$} \\
\hline & Media & $\mathrm{DE}$ & Media & $\mathrm{DE}$ & $\begin{array}{c}\text { Tamaño del } \\
\text { efecto (d) }\end{array}$ & Media & $\mathrm{DE}$ & Media & $\mathrm{DE}$ & \\
\hline Bienestar físico & 53,6 & 10 & 45,6 & 9,5 & 0,8 & 51,3 & 10 & 47,1 & 10,5 & 0,4 \\
\hline Bienestar emocional & 56 & 8,9 & 48,7 & 9,6 & 0,8 & 52,4 & 9,5 & 51,4 & 10,4 & 0,1 \\
\hline Estado de ánimo & 48,3 & 10,9 & 44,2 & 9,9 & 0,4 & 47,2 & 10,3 & 44,9 & 10,6 & 0,2 \\
\hline Autopercepción & 54,1 & 10,5 & 49,4 & 9,3 & 0,5 & 52,9 & 9,6 & 50,1 & 10,3 & 0,3 \\
\hline Autonomía & 53,1 & 10,4 & 49 & 10,3 & 0,4 & 51,8 & 10,2 & 49,8 & 10,8 & 0,2 \\
\hline Relación con los padres y vida familiar & 53,9 & 10 & 48,2 & 10,7 & 0,5 & 51,4 & 10,1 & 49,9 & 11,3 & 0,1 \\
\hline Apoyo social y relación con pares & 55,9 & 10,8 & 53,9 & 10,2 & 0,2 & 54,5 & 10,3 & 54,9 & 10,6 & 0 \\
\hline Ambiente escolar & 56,9 & 11,1 & 46,2 & 8,5 & 1,1 & 49,4 & 11 & 51,9 & 11 & $-0,2$ \\
\hline Aceptación social (bullying) & 44,7 & 11,6 & 46 & 10,2 & $-0,1$ & 45,3 & 10,7 & 45,5 & 10,9 & 0 \\
\hline Recursos económicos & 47,9 & 9,7 & 47,8 & 8,7 & 0 & 48,4 & 9 & 47,3 & 9,2 & 0,1 \\
\hline
\end{tabular}


bargo, en un estudio con el propósito de analizar propiedades métricas de una medida no es tan importante la representatividad, sino la variabilidad que puedan aportar los casos para que sea posible obtener amplios recorridos en las puntuaciones, es decir, que la muestra esté compuesta de individuos sensibles de puntuar a lo largo de toda la escala de valores de las dimensiones estudiadas. La muestra de este estudio fue aleatoria e incluyó niños y adolescentes de ambos sexos de escuelas públicas y privadas de toda una ciudad de nuestro país. Por otra parte, este estudio no proporciona información acerca del comportamiento del cuestionario en población no escolarizada.

Otros estudios deberán proporcionar más evidencias sobre la fiabilidad y validez de la versión argentina del KIDSCREEN, ya que son numerosas las propiedades métricas que pueden pretenderse para una medida de este tipo, como la estabilidad prueba-reprueba de la medida o la validez convergente y discriminante con instrumentos similares o con otras medidas, tales como las de salud mental o de discapacidad y su sensibilidad a los cambios para detectar efectos de tratamientos o intervenciones preventivas. Este instrumento es útil para propósitos diversos, como establecer estados de salud de niños y adolescentes, evaluar servicios sociales o sanitarios de actuación preventiva o terapéutica, así como discriminar el impacto de diferentes situaciones vitales sobre la salud, teniendo como referencia los datos de esta muestra de la ciudad de Bahía Blanca, así como los valores proporcionados por las versiones originales de trece países europeos con los cuales se podrán establecer comparaciones.

\section{CONCLUSIÓN}

Este estudio muestra que la versión argentina del KIDSCREEN-52 presenta una aceptable fiabilidad y validez para ser utilizado en investigaciones sobre la CVRS de la población argentina de 8 a 18 años.

\section{Agradecimientos}

A la Magister Silvina Spagnolo, la Magister Paula Abrego y la Médica Lilian Elosegui, de Epidemiología Ambiental de la Municipalidad de Bahía Blanca, por su meritoria labor en el trabajo de campo del estudio.

\section{BIBLIOGRAFÍA}

1. Testa MA, Simonson DC. Assessment of quality of life outcomes. N Engl J Med 1996;334:835-40.
2. PatrickD, Erickson P. Health Policy, Quality of Life: Health Care Evaluation and Resource Allocation. Nueva York: Oxford University Press; 1993.

3. Békési A, Török S, Kökönyei G, Bokrétás I, Szentes A, Telepóczki G and The European KIDSCREEN Group. Health-related quality of life changes of children and adolescents with chronic disease after participation in therapeutic recreation camping program. Health and Quality of Life Outcomes. 2011;9(43). [Acceso: febrero 2012]. Disponible en: http://www.hqlo.com/content/9/1/43.

4. Detmar SB, Bruil J, Ravens-Sieberer U, Gosch A, Bisegger $C$; the European KIDSCREEN group. The Use of Focus Groups in the Development of the KIDSCREEN HRQL Questionnaire. Qual Life Res 2006;15(8):1345-53.

5. Solans M, Pane S, Estrada MD, Serra-Sutton V, Berra S, Herdman M, Alonso J, Rajmil L. Health-related quality of life measurement in children and adolescents: a systematic review of generic and disease-specific instruments. Value Health 2008;11(4):742-64.

6. Bibliopro: Biblioteca virtual de Resultados Percibidos por los Pacientes (PRO - Patient Reported Outcomes). Disponible en: http://bibliopro.imim.es

7. Rajmil L, Roizen M, Urzúa A, Hidalgo-Rasmussen C, et al; Working Group on HRQOL in Children in Ibero-American Countries. Health-Related Quality of Life Measurement in Children and Adolescents in Ibero-American Countries, 2000 to 2010. Value Health 2012;15(2):321-22.

8. Moroldo MB, De Cunto C, Hübscher O, Liberatore D, et al. Cross-cultural adaptation and validation of an Argentine Spanish Version of the Stanford Childhood Health Assessment Questionnaire. Arthritis Care Res 1998;11(5):382-90.

9. Roizen M, Rodríguez S, Bauer G, Medin G, et al. Initial validation of the Argentinean Spanish version of the PedsQL 4.0 Generic Core Scales in children and adolescents with chronic diseases: acceptability and comprehensibility in low-income settings. Health Qual Life Outcomes 2008;6:59.

10. Berra S, Bustingorry V, Henze C, Díaz MP, et al. Adaptación transcultural del cuestionario KIDSCREEN para medir la calidad de vida relacionada con la salud en población argentina de 8 a 18 años. Arch Argent Pediatr 2009;107(4):307-14.

11. The KIDSCREEN Group Europe. The KIDSCREEN Questionnaires. Quality of life questionnaires for children and adolescents. Lengerich: Pabst Science Publishers; 2006.

12. Scientific Advisory Committee of the Medical Outcomes Trust. Assessing health status and quality-of-life instruments: attributes and review criteria. Qual Life Res 2002;11:193-205.

13. Herdman M, Rajmil L, Ravens-Sieberer U, Bullinger M, Power M, Alonso J; European Kidscreen Group European Disabkids Group. Expert consensus in the development of a European health-related quality of life measure for children and adolescents: a Delphi study. Acta Paediatr 2002;91(12):1385-90.

14. Ravens-Sieberer U, Gosch A, Rajmil L, et al; KIDSCREEN Group. The KIDSCREEN-52 quality of life measure for children and adolescents: psychometric results from a crosscultural survey in 13 European countries. Value Health 2008;11(4):645-58.

15. Beaton DE, Bombardier C, Guillemin F, Ferraz MB. Guidelines for the process of cross-cultural adaptation of selfreport measures. Spine 2000;25:3186-91.

16. Currie $\mathrm{C}$, et al. Indicators of socioeconomic status for adolescents: the WHO Health Behaviour in School-aged Children survey. Health Educ Res 1997;12(3):385-97.

17. Currie CE, Elton RA, Todd J, Platt S. Indicators of socioeconomic status for adolescents: the WHO Health Behaviour in School-aged Children survey. Health Educ Res 
1997;12(3):385-97.

18. Cronbach JL. Coefficient alpha and internal structure of tests. Psychometrika 1951;16:297-334.

19. Nunnally JC, Bernstein IH. Psychomertric theory. 3rd ed. Nueva York: McGraw-Hill; 1994.

20. Embretson SE, ReiseSP. Item response theory for psychologists. Londres: Lawrence Erlbaum Associates, Inc.; 2000.

21. Streiner D, Norman G. Health Measurement Scales. A practical guide to their development and use. Nueva York: Oxford University Press; 1993.
22. Byrne MB. Structural equation modeling with LISREL, PRELIS, and SIMPLIS: basic concepts, applications and programming. Londres: Lawrence Erlbaum Associates; 1993.

23. Cohen J. Statistical power analysis for the behavioral sciences. 2nd ed. Hillsdale: Lawrence Erlbaum Associates, Inc.; 1998.

24. Tebe C, Berra S, Herdman M, Aymerich M, Alonso J, Rajmil L. Fiabilidad y validez de la versión española del KIDSCREEN-52 para población infantil y adolescente. Med Clin (Barc) 2008;130(17):650-4. 